\title{
Optimal Decay Rates for Kirchhoff Plates with Intermediate Damping
}

\author{
J. C. V. BRAVO ${ }^{*}$, H. P. OQUENDO ${ }^{2}$ and J. E. M. RIVERA ${ }^{3}$ \\ Received on May 6, 2019 / Accepted on February 13, 2020
}

\begin{abstract}
In this paper we study the asymptotic behavior of Kirchhoff plates with intermediate damping. The damping considered contemplates the frictional and the Kelvin-Voigt type dampings. We show that the semigroup those equations decays polynomially in time at least with the rate $t^{-1 /(2-2 \theta)}$, where $\theta$ is a parameter in the interval $[0,1[$. Moreover, we prove that this decay rate is optimal.
\end{abstract}

Keywords: plate equation, polynomial decay, optimal decay, frictional damping, Kelvin-Voigt type damping.

\section{INTRODUCTION}

Consider $\Omega$ a bounded open set of $\mathbb{R}^{n}$ with smooth boundary. This paper deals with the asymptotic stability of the solutions of the following Kirchhoff plate equation

$$
\left.u_{t t}-\gamma \Delta u_{t t}+\beta \Delta^{2} u+\varepsilon(-\Delta)^{\theta} u_{t}=0 \quad \text { in } \quad \Omega \times\right] 0, \infty[
$$

satisfying the boundary conditions

$$
u=0, \quad \Delta u=0 \quad \text { on } \quad \partial \Omega \times] 0, \infty[
$$

and the initial data

$$
u(x, 0)=u_{0}(x), u_{t}(x, 0)=u_{1}(x) \quad x \in \Omega .
$$

Here, the rotational inertia coefficient $\gamma$, the elasticity coefficient $\beta$ and the damping coefficient $\varepsilon$, are positive and the exponent $\theta$ is considered in the interval $[0,1]$. The term $(-\Delta)^{\theta} u_{t}$ in equation

*Corresponding author: Juan Carlos Vila Bravo - E-mail: jcvb@ufpr.br

${ }^{1}$ Department of Mathematics, Federal University of Paraná, Brazil - E-mail: jcvb@ufpr.br https://orcid.org/0000-00015277-3695

2 Department of Mathematics, Federal University of Paraná, Brazil - E-mail: higidio@ufpr.br https://orcid.org/00000001-6936-7183

${ }^{3}$ National Laboratory for Scientific Computation, Brazil - E-mail: rivera@lncc.br https://orcid.org/0000-0001-56952623 
(1.1) sets up an intermediate dissipation which includes the frictional damping $(\theta=0)$ and the Kelvin-Voigt damping $(\theta=1)$.

It is well-known that the semigroup of the system (1.1)-(1.3) is exponentially stable when the damping in equation (1.1) is of Kelvin-Voigt type and that this uniform decay is lost if the damping is of frictional type. We do not find in the literature results about the exact decay rates for the associated semigroups to Kirchhoff plates with intermediate damping between frictional dissipation and the Kelvin-Voigt type. This paper aims to find some answers related to this subject.

There exist many works about the stability of the solutions of plate models with some dissipative mechanisms. A variety of plate models can be found in the books $[7,9,10]$.

Concerning plate models with other dissipative mechanisms, we are going to mention some of them. In [2], Barbosa et al. studied the stability of the thermoelastic plate

$$
\begin{gathered}
\left.u_{t t}+\Delta^{2} u-M\left(\int_{\Omega}|\nabla u|^{2} d x\right)-\Delta u_{t t}+f(u)+v \Delta \theta=0, \quad \text { in } \quad \Omega \times\right] 0, \infty[, \\
\left.\theta_{t}-\omega \Delta \theta-(1-\omega) \int_{0}^{\infty} k(s) \Delta \theta(t-s) d s-v \Delta u_{t}=0, \quad \text { in } \quad \Omega \times\right] 0, \infty[,
\end{gathered}
$$

with boundary conditions $u=\Delta u=\theta=0$. They showed the existence of exponential attractors using semigroup theory.

In [13], the authors considered the viscoelastic plate.

$$
\left.u_{t t}-\gamma \Delta u_{t t}+\beta \Delta^{2} u-\int_{0}^{\infty} g(s) \Delta^{2 \theta} u(t-s) d s=0, \quad \text { in } \quad \Omega \times\right] 0, \infty[,
$$

with boundary conditions $u=\Delta u=0$ and exponentially decreasing kernel $g$. For regular initial data, it was shown that the associated semigroup is polynomially stable with decay rate $t^{-1 /(4-4 \theta)}$ when $\theta<1$.

For plates with boundary damping, we have the work of Rao et al. [14], who studied the stability of the equation

$$
\left.u_{t t}-\gamma \Delta u_{t t}+\Delta^{2} u=0, \quad \text { in } \quad \Omega \times\right] 0, \infty[,
$$

satisfying the boundary conditions

$$
\begin{aligned}
& \left.u=\frac{\partial u}{\partial v}=0, \quad \text { on } \quad \Gamma_{0} \times\right] 0, \infty[, \\
& \left.\Delta u+(1-\mu) B_{1} u+\eta=0, \quad \text { on } \quad \Gamma_{1} \times\right] 0, \infty[, \\
& \left.\frac{\partial \Delta u}{\partial v}+(1-\mu) \frac{\partial B_{2} u}{\partial \tau}-\gamma \frac{\partial u_{t t}}{\partial v}+\frac{\partial^{2} u_{t}}{\partial \tau^{2}}-\xi=0, \quad \text { on } \quad \Gamma_{1} \times\right] 0, \infty[.
\end{aligned}
$$

Here, $\Gamma_{0} \cup \Gamma_{1}=\partial \Omega$ and $\eta, \xi$ are solutions of the equations

$$
\begin{array}{rrrr}
\eta_{t}-\frac{\partial u_{t}}{\partial v}+\eta=0, & \text { on } & \left.\Gamma_{1} \times\right] 0, \infty[ \\
\xi_{t}-u_{t}+\xi=0, & \text { on } & \left.\Gamma_{1} \times\right] 0, \infty[.
\end{array}
$$


For any initial data, they showed the strong stability of the system. Moreover, for regular initial data and with boundary $\Gamma_{1}$ satisfying suitable geometric condition, they proved that the energy decays with polynomially rate $t^{-1}$.

Other authors studied the stability problem for plates with different dissipative mechanisms. the main results are related to the asymptotic stability of the semigroup with exponential or poly nomial decay. See for instance $[1,5,6,8,11,12,15,16]$.

It is well known that the operator $A=-\Delta$ defined in the space $D(A)=H^{2}(\Omega) \cap H_{0}^{1}(\Omega)$ is a positive self-adjoint operator in the Hilbert space $L^{2}(\Omega)$. Even more, this operator has compact inverse. Using this notation for the operator $-\Delta$ the system (1.1)-(1.3) can be written in the following abstract setting

$$
u_{t t}+\gamma A u_{t t}+\beta A^{2} u+\varepsilon A^{\theta} u_{t}=0
$$

satisfying the initial conditions

$$
u(0)=u_{0}, u_{t}(0)=u_{1}
$$

The main result of this paper deals with the asymptotic behavior of the semigroup for the abstract system (1.4)-(1.5). To be more precise, we show that the associated semigroup decays polynomially as the time goes to infinity with rate $t^{-1 /(2-2 \theta)}$ for regular initial data (see Theorem 2$)$. In this same Theorem we also show the optimality of this decay rate.

The remainder of this article is organized as follows: In section 2 we introduce the semigroup of the system (1.4)-(1.5). In section 3 we enunciate and prove the main result of this paper.

\section{EXISTENCE OF SOLUTIONS}

We are going to use the semigroup theory to show existence of solutions for the system of equations (1.4)-(1.5). It is important recalling that $A=-\Delta, D(A)=H^{2}(\Omega) \cap H_{0}^{1}(\Omega)$, is a positive selfadjoint operator with compact inverse in the Hilbert space $\mathbb{H}:=L^{2}(\Omega)$. Therefore, the operator $A^{\theta}$ is self-adjoint, positive for $\theta \in \mathbb{R}$ and the embedding

$$
D\left(A^{\theta_{1}}\right) \hookrightarrow D\left(A^{\theta_{2}}\right)
$$

is compact for $\theta_{1}>\theta_{2}$. Here, the norm in the space $D\left(A^{\theta}\right)$ is given by $\|u\|_{D\left(A^{\theta}\right)}:=\left\|A^{\theta} u\right\|$, where $\|\cdot\|$ denotes the norm of the Hilbert space $\mathbb{H}$. More details about fractional operators can be found in [4]. Note that, in view of the Riesz representation Theorem we have: for $v_{1}, v_{2} \in D\left(A^{1 / 2}\right)$,

$$
\left\langle(I+\gamma A) v_{1}, v_{2}\right\rangle=\left\langle v_{1}, v_{2}\right\rangle+\gamma\left\langle A^{1 / 2} v_{1}, A^{1 / 2} v_{2}\right\rangle,
$$

where $\langle\cdot, \cdot\rangle$ on the right side of this equation denotes the inner product in the space $\mathbb{H}$.

Now, if we consider the vector $U(t)=\left(u, u_{t}\right)$, then the system (1.4)-(1.5) can be written in an abstract framework as

$$
\frac{d}{d t} U(t)=\mathbb{B} U(t), \quad U(0)=U_{0},
$$


where $U_{0}=\left(u_{0}, u_{1}\right)$ and the operator $\mathbb{B}$ is given by

$$
\mathbb{B} U=\left(v,-(I+\gamma A)^{-1}\left\{\beta A^{2} u+\varepsilon A^{\theta} v\right\}\right),
$$

for $U=(u, v)$. To study the abstract system (2.2) through semigroup theory we are going to work in the Hilbert space

$$
\mathbb{X}=D(A) \times D\left(A^{1 / 2}\right),
$$

where the inner product is defined by

$$
\left\langle U_{1}, U_{2}\right\rangle_{\mathbb{X}}=\left\langle v_{1}, v_{2}\right\rangle+\gamma\left\langle A^{1 / 2} v_{1}, A^{1 / 2} v_{2}\right\rangle+\beta\left\langle A u_{1}, A u_{2}\right\rangle,
$$

for $U_{i}=\left(u_{i}, v_{i}\right), i=1,2$. Here, $\langle\cdot, \cdot\rangle$ on the right side of this equation denotes the inner product in the space $\mathbb{H}$. With theses considerations, the domain of the operator $\mathbb{B}$ is defined by

$$
D(\mathbb{B})=\left\{U \in \mathbb{X}: v \in D(A), u \in D\left(A^{3 / 2}\right)\right\} .
$$

To show the existence of solutions of the system (2.2) we invoke a result from Zheng-Liu' book [10]:

Theorem 1 (see Theorem 1.2.4 in [10]). Let $\mathbb{B}$ a linear operator with dense domain $D(\mathbb{B})$ in a Hilbert space $\mathbb{X}$. If $\mathbb{B}$ is dissipative and $0 \in \rho(\mathbb{B})$, the resolvent set of $\mathbb{B}$, then $\mathbb{B}$ is the generator of a $C_{0}$-semigroup of contractions on $\mathbb{X}$.

We are going to verify that the operator $\mathbb{B}$ defined by (2.3) satisfies the conditions of this theorem. From definition (2.4), we easily see that $D(\mathbb{B})$ is dense in $\mathbb{X}$. On the other hand, performing a simple computation we obtain

$$
\operatorname{Re}\langle\mathbb{B} U, U\rangle_{\mathbb{X}}=-\varepsilon\left\|A^{\frac{\theta}{2}} v\right\|^{2}, \quad \forall U \in D(\mathbb{B}),
$$

that is, the operator $\mathbb{B}$ is dissipative. To complete the conditions of the above theorem, it remains to show that $0 \in \rho(\mathbb{B})$. Let $F=(f, g) \in \mathbb{X}$, let us see that the stationary problem $\mathbb{B} U=F$ has a solution $U=(u, v)$. From definition of the operator $\mathbb{B}$ in (2.3), this system can be written by

$$
v=f, \quad \beta A^{2} u+\varepsilon A^{\theta} v=-(I+\gamma A) g .
$$

This problem can be put in a variational formulation: to find $u \in D(A)$ such that

$$
\beta\langle A u, A z\rangle=-\langle g,(I+\gamma A) z\rangle-\varepsilon\left\langle f, A^{\theta} z\right\rangle, \quad \forall z \in D(A) .
$$

Now, applying the Lax-Milgram Theorem we have a unique solution $U \in \mathbb{H}$. As this solution satisfies (2.6) we can conclude that $U \in D(\mathbb{B})$. Moreover, taking $z=u$ in (2.7) and applying Cauchy-Schwarz and Young inequalities we conclude that

$$
\|A u\|^{2} \leq C\|F\|_{\mathbb{X}}^{2}
$$


This inequality and the first equation of (2.6) imply that $\|U\|_{\mathbb{X}} \leq C\|F\|_{\mathbb{X}}$, so we have 0 belongs to the resolvent set $\rho(\mathbb{B})$. Consequently, from Theorem 1 we have $\mathbb{B}$ is the generator of a contractions semigroup.

Finally, the well-posedness of the system (1.4)-(1.5) is a consequence of the semigroup theory. We state this result in the following theorem

Theorem 2. For $U_{0}=\left(u_{0}, u_{1}\right) \in D(\mathbb{B})$ there exist a unique solution of the system (1.4)-(1.5) in the space

$$
U=\left(u, u_{t}\right) \in C\left(\left[0, \infty[; D(\mathbb{B})) \cap C^{1}([0, \infty[; \mathbb{X})\right.\right.
$$

\section{STABILITY RESULTS}

In this section we study the asymptotic behavior of the semigroup $e^{t \mathbb{B}}$ associated with the system (1.4)-(1.5). To do this, we are going to use the following spectral characterization for the polynomial stability of semigroups due to Borichev and Tomilov:

Theorem 1 (see [3]). Let $\mathbb{B}$ be the generator of a $C_{0}$-semigroup of bounded operators on a Hilbert space such that $i \mathbb{R} \subset \rho(\mathbb{B})$. Then we have

$$
\left\|e^{t \mathbb{B}} U_{0}\right\|_{\mathbb{X}} \leq C t^{-1 / \alpha}\left\|U_{0}\right\|_{D(\mathbb{B})}, \quad \forall t>0, U_{0} \in D(\mathbb{B})
$$

if and only if,

$$
\limsup _{|\lambda| \rightarrow \infty}|\lambda|^{-\alpha}\left\|(i \lambda I-\mathbb{B})^{-1}\right\|<\infty
$$

Let $\lambda \in \mathbb{R}$ and $F=(f, g) \in \mathbb{X}$. In what follows, the stationary problem $(i \lambda-\mathbb{B}) U=F$ will be considered several times in the course of this article. Note that $U=(u, v)$ is a solution of this problem if the following equations are satisfied:

$$
i \lambda u-v=f, \quad i \lambda(1+\gamma A) v+\beta A^{2} u+\varepsilon A^{\theta} v=(1+\gamma A) g .
$$

In what follows, $C$ denotes a positive constant that assume different values in different places. The main result of this paper is given by the following theorem.

Theorem 2. Consider the semigroup of the system (1.4)-(1.5). For $\theta \in[0,1[$ we have the following polynomial decay

$$
\left\|e^{t \mathbb{B}} U_{0}\right\|_{\mathbb{X}}<C t^{-1 /(2-2 \theta)}\left\|U_{0}\right\|_{D(\mathbb{B})}, \quad \forall t>0 \text { and } U_{0} \in D(\mathbb{B}) .
$$

Moreover, this decay rate is optimal in the following sense: it does not decay with the rate $t^{-\kappa}$, for $\kappa>1 /(2-2 \theta)$. 
Proof. We are going to use Theorem 1 to show this theorem. Since $0 \in \rho(\mathbb{B})$ there exists $\delta>0$ such that $]-i \delta, i \delta[\subset \rho(\mathbb{B})$, so to show that $\rho(\mathbb{B})$ contains the imaginary axis is sufficed to show that $i \lambda \in \rho(\mathbb{B})$ for real $\lambda$ such that $|\lambda| \geq \delta$. Simultaneously we are going to prove the estimative $\|U\|_{\mathbb{X}} \leq C|\lambda|^{2-2 \theta}\|F\|_{\mathbb{X}}$ for the solution $U$ of the stationary equation $(i \lambda-\mathbb{B}) U=F$.

Note that, from identity (2.5) we easily obtain

$$
\varepsilon\left\|A^{\frac{\theta}{2}} v\right\|^{2}=\operatorname{Re}\langle(i \lambda-\mathbb{B}) U, U\rangle_{\mathbb{X}}=\operatorname{Re}\langle F, U\rangle_{\mathbb{X}} \leq\|F\|_{\mathbb{X}}\|U\|_{\mathbb{X}}
$$

Applying the duality product to the second equation of (3.1) with $A^{\theta} u$ and taking into account that the fractional powers of the operator $A$ are self-adjoint, we obtain

$$
\begin{aligned}
\beta\left\|A^{\frac{\theta+2}{2}} u\right\|^{2}= & \left\langle i \lambda A^{\frac{\theta}{2}} v, A^{\frac{\theta}{2}} u\right\rangle+\gamma\left\langle i \lambda A^{\frac{\theta}{2}} v, A^{\frac{\theta+2}{2}} u\right\rangle-\varepsilon\left\langle A^{\frac{3 \theta-2}{2}} v, A^{\frac{\theta+2}{2}} u\right\rangle \\
& +\left\langle A^{\frac{\theta}{2}} f, A^{\frac{\theta}{2}} u\right\rangle+\gamma\left\langle A^{\frac{\theta}{2}} f, A^{\frac{\theta+2}{2}} u\right\rangle .
\end{aligned}
$$

Applying the Cauchy-Schwarz inequality, Young inequality $a b \leq \delta a^{2}+(4 \delta)^{-1} b^{2}, \delta>0$ and taking into account that $\frac{3 \theta-2}{2}<\frac{\theta}{2}$ we get that there exists a positive constant $C$ such that

$$
\beta\left\|A^{\frac{\theta+2}{2}} u\right\|^{2} \leq C\left\{\left(1+\lambda^{2}\right)\left\|A^{\frac{\theta}{2}} v\right\|^{2}+\|F\|_{\mathbb{X}}^{2}\right\} .
$$

On the other hand, from the first equation of (3.1), we have

$$
\lambda^{2}\left\|A^{\frac{\theta}{2}} u\right\|^{2} \leq 2\left(\left\|A^{\frac{\theta}{2}} v\right\|^{2}+\|F\|_{\mathbb{X}}^{2}\right) .
$$

Now we are going to use an interpolation inequality. Since

$$
1=\delta\left(\frac{\theta+2}{2}\right)+(1-\delta) \frac{\theta}{2}, \quad \text { for } \quad \delta=\frac{2-\theta}{2},
$$

using inequalities (3.3) and (3.4) we get that

$$
\begin{aligned}
\|A u\|^{2} & \leq C\left\|A^{\frac{\theta+2}{2}} u\right\|^{2 \delta}\left\|A^{\frac{\theta}{2}} u\right\|^{2(1-\delta)} \\
& \leq C\left\{\left(1+\lambda^{2}\right)\left(\left\|A^{\frac{\theta}{2}} v\right\|^{2}+\|F\|_{\mathbb{X}}^{2}\right)\right\}^{\frac{2-\theta}{2}}\left\{\lambda^{-2}\left(\left\|A^{\frac{\theta}{2}} v\right\|^{2}+\|F\|_{\mathbb{X}}^{2}\right)\right\}^{\frac{\theta}{2}} .
\end{aligned}
$$

Therefore, in view of inequality (3.2), we conclude that

$$
\|A u\|^{2} \leq C\left(1+\lambda^{2-2 \theta}\right)\left\{\|F\|_{\mathbb{X}}\|U\|_{\mathbb{X}}+\|F\|_{\mathbb{X}}^{2}\right\}
$$

for $|\lambda| \geq \delta$. On the other hand, applying the duality product to the second equation of (3.1) with $u$ and using the first equation we have

$$
\|v\|^{2}+\gamma\left\|A^{\frac{1}{2}} v\right\|^{2}=\beta\|A u\|^{2}+\varepsilon\left\langle v, A^{\theta} u\right\rangle-\langle f, u\rangle-\gamma\langle f, A u\rangle-\langle v, f\rangle-\gamma\langle v, A f\rangle .
$$

Applying the Cauchy-Schwarz and Young inequalities, and using the estimates (3.5), we obtain

$$
\left\|A^{\frac{1}{2}} v\right\|^{2} \leq C\left(1+\lambda^{2-2 \theta}\right)\left\{\|F\|_{\mathbb{X}}\|U\|_{\mathbb{X}}+\|F\|_{\mathbb{X}}^{2}\right\} .
$$


Finally, from this inequality and (3.5) we can conclude that

$$
\|U\|_{\mathbb{X}}^{2} \leq C\left(1+\lambda^{2-2 \theta}\right)\left\{\|F\|_{\mathbb{X}}\|U\|_{\mathbb{X}}+\|F\|_{\mathbb{X}}^{2}\right\} .
$$

As $|\lambda| \geq \delta$ we have $\|U\|_{\mathbb{X}}^{2} \leq C \lambda^{2-2 \theta}\left\{\|F\|_{\mathbb{X}}\|U\|_{\mathbb{X}}+\|F\|_{\mathbb{X}}^{2}\right\}$ from where follows the desired inequality $\|U\|_{\mathbb{X}} \leq C \lambda^{2-2 \theta}\|F\|_{\mathbb{X}}$. Note that, if $F=0$ then $U=0$, consequently $\mathbb{B}$ does not have eigenvalues in the imaginary axis. As the operator $\mathbb{B}^{-1}$ is compact its spectrum is constituted only by eigenvalues, so $i \mathbb{R} \subset \rho(\mathbb{B})$. This completes the proof of the polynomial decay of the semigroup with rate $t^{-1 /(2-2 \theta)}$.

Optimality of the decay rate: Since $A$ is a positive self-adjoint operator with compact resolvent, its spectrum is constituted by positive eigenvalues $\alpha_{n}, n \in \mathbb{N}$, with $\alpha_{n} \rightarrow \infty$. Let us denote by $\left(e_{n}\right)$ the corresponding eigenvectors, that is

$$
A e_{n}=\alpha_{n} e_{n}, \quad n \in \mathbb{N} .
$$

We consider $F_{n}=\left(0,-\tilde{e}_{n}\right) \in \mathbb{X}$ where $\tilde{e}_{n}=e_{n} /\left\|A^{1 / 2} e_{n}\right\|$, then the solution $U=(u, v)$ of the system $(i \lambda I-\mathbb{B}) U=F_{n}$ satisfies the following conditions:

$$
v=i \lambda u, \quad i \lambda v+(I+\gamma A)^{-1}\left\{\beta A^{2} u+\varepsilon A^{\theta} v\right\}=-\tilde{e}_{n} .
$$

By substituting the first identity in the second equation we obtain

$$
\lambda^{2}(I+\gamma A) u-\beta A^{2} u-i \varepsilon \lambda A^{\theta} u=(I+\gamma A) \tilde{e}_{n} .
$$

Now, we are going to look for by solutions of the form $u=\eta \tilde{e}_{n}$ for some complex number $\eta$. Therefore, the coefficient $\eta$ must satisfy the equation

$$
\lambda^{2}\left(1+\gamma \alpha_{n}\right) \eta-\beta \alpha_{n}^{2} \eta-i \varepsilon \lambda \alpha_{n}^{\theta} \eta=\left(1+\gamma \alpha_{n}\right)
$$

Solving this equation we have

$$
\eta=\frac{1+\gamma \alpha_{n}}{\lambda^{2}\left(1+\gamma \alpha_{n}\right)-\beta \alpha_{n}^{2}-i \varepsilon \lambda \alpha_{n}^{\theta}} .
$$

In this point, taking

$$
\lambda=\lambda_{n}:=\frac{\sqrt{\beta} \alpha_{n}}{\sqrt{1+\gamma \alpha_{n}}}
$$

the above formula becomes

$$
\eta=\eta_{n}=i \frac{1+\gamma \alpha_{n}}{\varepsilon \lambda_{n} \alpha_{n}^{\theta}}
$$

If we introduce the notation $x_{n} \approx y_{n}$ meaning $\lim _{n \rightarrow \infty} \frac{\left|x_{n}\right|}{\left|y_{n}\right|}$ is a positive real number, then from (3.6) and (3.7) we can assert that $\lambda_{n} \approx \alpha_{n}^{1 / 2}$ and $\eta_{n} \approx \lambda_{n}^{1-2 \theta}$. Therefore, if $U_{n}=\left(u_{n}, v_{n}\right)$ is the solution of the system $\left(i \lambda_{n}-A\right) U=F_{n}$, we obtain

$$
\left\|A^{1 / 2} v_{n}\right\|=\lambda_{n}\left\|A^{1 / 2} u_{n}\right\|=\lambda_{n}\left|\eta_{n}\right|\left\|A^{1 / 2} \tilde{e}_{n}\right\| \geq \delta \lambda_{n}^{2-2 \theta},
$$


for some $\delta>0$ and $n$ large enough. From this estimate, we conclude that

$$
\left\|U_{n}\right\|_{\mathbb{X}} \geq \gamma\left\|A^{1 / 2} v_{n}\right\| \geq \gamma \delta \lambda_{n}^{2-2 \theta}
$$

Finally, let us suppose the semigroup decays with the rate $t^{-\kappa}$ for some $\kappa>1 /(2-2 \theta)$. From Theorem 1 we have $\lambda_{n}^{-1 / \kappa}\left\|U_{n}\right\|_{\mathbb{X}}$ is bounded. On the other hand, the above inequality implies that

$$
\lambda_{n}^{-1 / \kappa}\left\|U_{n}\right\|_{\mathbb{X}} \geq \gamma \delta \lambda_{n}^{2-2 \theta-\frac{1}{\kappa}} \rightarrow \infty,
$$

which is absurd. Therefore the decay rate $t^{-1 /(2-2 \theta)}$ is optimal.

RESUMO. Neste trabalho estudamos o comportamento assintótico das placas de Kirchhoff com amortecimento intermediário. O amortecimento considerado contempla as dissipações de tipo friccional e de Kelvin-Voigt. Mostramos que o semigrupo dessas equações decai polinomialmente no tempo com a taxa $t^{-1 /(2-2 \theta)}$, onde $\theta$ é um parâmetro no intervalo $[0,1[$. Além disso, provamos que a taxa de decaimento encontrada é ótima.

Palavras-chave: equação de placa, decaimento polinomial, decaimento ótimo, amortecimento friccional, dissipação de tipo Kelvin-Voigt.

\section{REFERENCES}

[1] K. Ammari, M. Dimassi \& M. Zerzeri. Rate of decay of some abstract Petrowsky-like dissipative semi-groups. Semigroup Forum 93 (2016), 1-16.

[2] A.R.A. Barbosa \& T.F. Ma. Long-time dynamics of an extensible plate equation with thermal memory. J. Math. Anal. Appl. 416 (2014), 143-165.

[3] A. Borichev \& Y. Tomilov. Optimal polynomial decay of functions and operator semigroups. Math. Ann. 347 (2010), 455-478.

[4] K.J. Engel \& R. Nagel. “One-parameter semigroups for linear evolution equations”. Springer 2000.

[5] F. Franchi, B. Lazzari \& R. Nibbi. On the asymptotic stability for Kirchhoff plates with viscoelastic dissipation. Meccanica 53 (2018), 295-304.

[6] M. Grasselli \& M. Squassina. Exponential stability and singular limit for a linear thermoelastic plate with memory effects. Adv. Math. Sci. Appl. 16 (2006), 15-31.

[7] V. Komornik. "Exact controllability and stabilization. The multiplier method. RAM: Research in Applied Mathematics". Masson, Paris; John Wiley \& Sons, 1994.

[8] I. Lasiecka \& R. Triggiani. Exact controllability and uniform stabilization of Kirchhoff plates with boundary control only on $\left.\Delta w\right|_{\Sigma}$ and homogeneous boundary displacement. J. Differential Equations 93 (1991), 62-101.

[9] J. Lagnese. Boundary stabilization of thin plates. SIAM Studies in Applied Mathematics 10. Philadelphia, PA, 1989. 
[10] Z. Liu \& S. Zheng. "Semigroups associated with dissipative systems". Chapman \& Hall, 1999.

[11] M.I. Mustafa \& M. Kafini. Decay rates for memory-type plate system with delay and source term. Math. Methods Appl. Sci. 40 (2017), 883-895.

[12] H.P. Oquendo. Uniform decay for a plate equation partially dissipative. Appl. Math. Comput. 146 (2003), 121-134.

[13] H.P. Oquendo \& M. Astudillo. Optimal decay for plates with rotational inertia and memory. Preprint.

[14] B. Rao \& A. Wehbe. Polynomial energy decay rate and strong stability of Kirchhoff plates with non-compact resolvent. J. Evol. Equ. 5 (2005), 137-152.

[15] J.E.M. Rivera, H.P. Oquendo \& M.L. Santos. Asymptotic behavior to a von Kármán plate with boundary memory conditions. Nonlinear Analysis 6 (2005) 1183-1205.

[16] M.A.J. Silva, J.E.M. Rivera \& R. Racke. On a class of nonlinear viscoelastic Kirchhoff plates: wellposedness and general decay rates. Appl. Math. Optim. 73 (2016), 165-194. 